\title{
THE COMPRESSIONAL MODULUS OF A MATERIAL PERMEATED BY A RANDOM DISTRIBUTION OF CIRCULAR CRACKS*
}

\author{
BY \\ H. D. GARBIN** AND L. KNOPOFF \\ University of California, Los Angeles
}

I. Introduction. $\mathrm{Wu}[1]$ has considered the problem of the elastic properties of a composite solid permeated by a sparse random distribution of identical spheriodal inclusions, treated by the methods of static elasticity. Limits can be taken to give the cases of included needles, spheres and disks. The properties of a spheroid are of course characterized by the smoothness and convexity of the surface. In the case of the disks, the singularities in stress at the edges are sufficiently great that, in this limit, a nonphysical result is obtained for the case in which the shear modulus of the obstacles is allowed to vanish. Wu was careful not to take this limit. Walsh [2], however, did take the disk limit for vanishing shear modulus of inclusions and attempted to apply the result to the long-wavelength scattering problem. Walsh [3] later recognized the difficulty and proceeded to avoid the problem of the singularities at the edges by retaining a nonzero aspect ratio to the crack.

Mal and Knopoff [4] considered the scattering at long wavelengths from spherical inhomogeneities and showed that the result was completely consistent with the static result. They also investigated the effect of a statistical distribution of radii of the spatially sparsely and randomly distributed spheres.

We consider the problem of the average elastic properties of a homogeneous elastic solid permeated by a sparse distribution of randomly located, randomly oriented thin circular obstacles in a scattering process. The boundary conditions on the two walls of the obstacles separately can either be free-free, in which the normal components of the stresses vanish, or liquid-elastic, in which the normal shear component of the stress vanishes while the normal compressional component of the stress and the normal component of the displacement are continuous. We can also make the liquid filler viscous by the introduction of impedance boundary conditions. The free-free and liquid-elastic problems will be considered in separate papers. We consider only the long-wavelength limit of the scattering problem. We assume, based on the demonstration for spheres, that this will also give the static result.

These problems may be considered to be models of a completely flawed object. Consider, for example, a building brick permeated with fine circular cracks, or the partial melting of a solid in which the mode of melting is along grain boundaries. In either of these problems, we might imagine that it would be of interest to learn something

* Received March 6, 1972.

** Now at Sandia Laboratories, Albuquerque, N. M. 87115 
about the concentration of inhomogencities without a microscopic examination of the interior.

Some progress on the attack of the problems of scattering of elastic waves by finite inclusions with sharp edges was made by Ang and Knopoff [5, 6] who obtained the solution to the two-dimensional cases of scattering by a single finite crack or strip. The problem, as in the present case, involves the solution of coupled dual singular integral equations. Mal et al. [7] have considered the scattering of normally incident compressional waves by a penny-shaped crack. The plan of this paper involves the solution of the problem of the scattering of long-wavelength compressional waves incident obliquely on a single circular crack imbedded in an infinite, elastic, homogeneous, isotropic medium. We then give a method for determining the properties of a medium randomly permeated by such cracks.

II. The integral equations. Let the location of the crack be given by $z=0,0 \leq$ $r<1$, in cylindrical coordinates $(r, \phi, z)$. All lengths are normalized with respect to the radius of the crack. The time harmonic behavior exp $(-i \omega t)$ in all the physical quantities is suppressed. The displacement vector $\mathbf{u}$ for the diffracted field satisfies

$$
k_{1}^{-2} \nabla(\nabla \cdot \mathfrak{u})-k_{2}^{-2} \nabla \times \nabla \times \mathfrak{u}+\mathfrak{u}=0,
$$

where

$$
k_{i}=\omega / v_{i}, \quad i=1,2
$$

and $v_{1}$ and $v_{2}$ are the compressional and shear wave velocities in the medium defined by the relations

$$
\begin{aligned}
& v_{1}^{2}=(\lambda+2 \mu) / \rho, \\
& v_{2}^{2}=\mu / \rho .
\end{aligned}
$$

$\lambda$ and $\mu$ are the usual Lamé elastic parameters and $\rho$ is the density.

Let the vector displacement field be decomposed into three scalar potentials $\Phi$, $\Psi$ and $\chi$ as

$$
\begin{aligned}
& u_{r}=\frac{\partial \Phi}{\partial r}+\frac{1}{r} \frac{\partial \Psi}{\partial \phi}+\frac{\partial^{2} \chi}{\partial z \partial r} \\
& u_{\phi}=\frac{1}{r} \frac{\partial \Phi}{\partial \phi}-\frac{\partial \Psi}{\partial r}+\frac{1}{r} \frac{\partial^{2} \chi}{\partial \phi \partial z} \\
& u_{s}=\frac{\partial \Phi}{\partial z}+\left(\frac{\partial^{2}}{\partial z^{2}}+k_{2}^{2}\right) \chi
\end{aligned}
$$

where $\Phi, \Psi$ and $\chi$ satisfy

$$
\nabla^{2} \Phi+k_{1}^{2} \Phi=0, \quad \nabla^{2} \Psi+k_{2}^{2} \Psi=0, \quad \nabla^{2} \chi+k_{2}^{2} \chi=0 . \quad(5 \mathrm{a}, \mathrm{b}, \mathrm{c})
$$

The normal components of the stress in terms of these potentials are

$$
\begin{aligned}
& \tau_{z r} / \mu=2 \frac{\partial^{2} \Phi}{\partial r \partial z}+\frac{1}{r} \frac{\partial^{2} \Psi}{\partial \phi} \frac{\partial}{\partial z}+\frac{\partial}{\partial r}\left(2 \frac{\partial^{2}}{\partial z^{2}}+k_{2}^{2}\right) \chi, \\
& \tau_{z \phi} / \mu=\frac{2}{r} \frac{\partial^{2} \Phi}{\partial \phi} \partial z-\frac{\partial^{2} \Psi}{\partial r \partial z}+\frac{1}{r} \frac{\partial}{\partial \phi}\left(2 \frac{\partial^{2}}{\partial z^{2}}+k_{2}^{2}\right) \chi,
\end{aligned}
$$




$$
\tau_{z z} / \mu=\left(2 k_{1}^{2}-k_{2}^{2}+2 \frac{\partial^{2}}{\partial z^{2}}\right) \Phi+2 \frac{\partial}{\partial z}\left(\frac{\partial^{2}}{\partial z^{2}}+k_{2}^{2}\right) \chi .
$$

Assume solutions of (5) to be

$$
\begin{aligned}
& \Phi(r, \phi, z)=\sum_{n=-\infty}^{\infty} e^{i n \phi} \int_{0}^{\infty}\left\{P_{1 n}(\zeta) \mp P_{2 n}(\zeta)\right\} J_{n}(\zeta r) e^{-\eta_{1}|z|} d \zeta, \\
& \Psi(r, \phi, z)=\sum_{n=-\infty}^{\infty} e^{i n \phi} \int_{0}^{\infty}\left\{Q_{1 n}(\zeta) \mp Q_{2 n}(\zeta)\right\} J_{n}(\zeta r) e^{-\gamma_{z}|z|} d \zeta, \\
& \chi(r, \phi, z)=\sum_{n=-\infty}^{\infty} e^{i n \phi} \int_{0}^{\infty}\left\{R_{1 n}(\zeta) \mp R_{2 n}(\zeta)\right\} J_{n}(\zeta r) e^{-\eta_{z}|z|} d \zeta,
\end{aligned}
$$

where

$$
\nu_{i}^{2}=\zeta^{2}-k_{i}^{2}, \quad \operatorname{Re} \nu_{i}>0, \quad i=1,2 .
$$

The $\mp$ sign in (7) refers to $z \gtrless 0$ respectively. The six functions $P_{1 n}(\zeta)$, etc. are determined from the boundary conditions. For the free-free problem,

$$
\tau_{z i}^{0}+\left.\tau_{z i}\right|_{2 \rightarrow 0^{ \pm}}=0 \quad r<1, \quad i=r, \phi, z,
$$

where the stresses $\tau^{0}$ refer to the incident field.

The displacement is continuous for $r>1$ and the stresses are continuous for all $r$. The latter condition implies

$$
\begin{gathered}
\int_{0}^{\infty}\left\{\left(2 \zeta^{2}-k_{2}^{2}\right) P_{2 n}(\zeta)+2 \nu_{2} \zeta^{2} R_{1 n}(\zeta)\right\} J_{n}(\zeta r) d \zeta=0 \\
\int_{0}^{\infty} \zeta\left[2 \nu_{1} P_{1 n}(\zeta)+\left(2 \zeta^{2}-k_{2}^{2}\right) R_{2 n}(\zeta)\right] J_{n}^{\prime}(\zeta r) d \zeta+\frac{i n}{r} \int_{0}^{\infty} \nu_{2} Q_{1 n}(\zeta) J_{n}(\zeta r) d \zeta=0 \\
\frac{i n}{r} \int_{0}^{\infty}\left[2 \nu_{1} P_{1 n}(\zeta)+\left(2 \zeta^{2}-k_{2}^{2}\right) R_{2 n}(\zeta)\right] J_{n}(\zeta r) d \zeta-\int_{0}^{\infty} \zeta \nu_{2} Q_{1 n}(\zeta) J_{n}^{\prime}(\zeta r) d \zeta=0
\end{gathered}
$$

Using the identity for Bessel functions

$$
x J_{n}^{\prime}(x) \pm n J_{n}(x)= \pm J_{n \mp 1}(x),
$$

(10b) and (10c) may be written as

$$
\int_{0}^{\infty}\left\{i\left[2 \nu_{1} P_{1 n}(\zeta)+\left(2 \zeta^{2}-k_{2}^{2}\right) R_{2 n}(\zeta)\right] \mp \nu_{2} Q_{1 n}(\zeta)\right\} \zeta r J_{n \mp 1}(\zeta r)=0 .
$$

A sufficient condition for this equation and (10a) to hold is

$$
\begin{gathered}
\left(2 \zeta^{2}-k_{2}^{2}\right) P_{2 n}(\zeta)+2 \nu_{2} \zeta^{2} R_{1 n}(\zeta)=0 \\
2 \nu_{1} P_{1 n}(\zeta)+\left(2 \zeta^{2}-k_{2}^{2}\right) R_{2 n}(\zeta)=0, \\
Q_{1 n}(\zeta)=0 .
\end{gathered}
$$

The continuity of the displacement for $r>1$ implies from (4) and (7)

$$
\int_{0}^{\infty} \zeta\left[P_{2 n}(\zeta)+v_{2} R_{1 n}(\zeta)\right] J_{n}^{\prime}(\zeta r) d \zeta+\frac{i n}{r} \int_{0}^{\infty} Q_{2 n}(\zeta) J_{n}(\zeta r) d \zeta=0, \quad r>1,
$$




$$
\begin{gathered}
\frac{i n}{r} \int_{0}^{\infty}\left[P_{2 n}(\zeta)+\nu_{2} R_{1 n}(\zeta)\right] J_{n}(\zeta r) d \zeta-\int_{0}^{\infty} \zeta Q_{2 n}(\zeta) J_{n}^{\prime}(\zeta r) d \zeta=0 \\
\int_{0}^{\infty}\left[\nu_{1} P_{1 n}(\zeta)+\zeta^{2} R_{2 n}(\zeta)\right] J_{n}(\zeta r) d \zeta=0
\end{gathered}
$$

There are three more relationships which arise from the boundary conditions (9), but these will require that incident waves be specified. We assume that a harmonic plane $P$-wave of frequency $\omega$ travels in the $x-z$ plane and strikes the crack obliquely. The incident wave is given by

$$
\begin{aligned}
\Psi^{0} & =\chi^{0}=0, \\
\Phi^{0} & =\exp (i(\alpha x+\beta z))=\exp (i \beta z) \sum_{n=-\infty}^{\infty} i^{n} \exp (i n \phi) J_{n}(\alpha r), \\
\alpha^{2}+\beta^{2} & =k_{1}^{2}
\end{aligned}
$$

Using (6), (7), (12), (14) and the identity (11) in Eqs. (9), we get

$$
\begin{aligned}
& \int_{0}^{\infty} G(\zeta) P_{1 n}(\zeta) J_{n}(\zeta r) d \zeta=\left(2 \alpha^{2}-k_{2}^{2}\right) i^{n} J_{n}(\alpha r), \quad r<1 \\
& \int_{0}^{\infty} d \zeta\left[ \pm i G(\zeta) R_{1 n}(\zeta)+\nu_{2} Q_{2 n}(\zeta)\right] J_{n \mp 1}(\zeta r)=\mp 2 \imath^{n} \beta J_{n \mp 1}(\alpha r)
\end{aligned}
$$

where $G(\zeta)=\left[4 \zeta^{2} \nu_{1} \nu_{2}-\left(2 \zeta^{2}-k_{2}^{2}\right)^{2}\right] /\left(2 \zeta^{2}-k_{2}^{2}\right)$. Putting (12) into (13) and using the identity (11), we obtain the following dual integral equations:

$$
\begin{array}{cc}
\int_{0}^{\infty} G(\zeta) P_{1 n}(\zeta) J_{n}(\zeta r) d \zeta=\left(2 \alpha^{2}-k_{2}^{2}\right) i^{n} J_{n}(\alpha r), \quad 0 \leq r<1 \\
\int_{0}^{\infty} \frac{\nu_{1} P_{1 n}(\zeta)}{2 \zeta^{2}-k_{2}^{2}} J_{n}(\zeta r) d \zeta=0, \quad r>1, \\
\int_{0}^{\infty} d \zeta \zeta\left[i G(\zeta) R_{1 n}(\zeta)+\nu_{2} Q_{2 n}(\zeta)\right] J_{n-1}(\zeta r)=-2 \alpha \beta i^{n} J_{n-1}(\alpha r), \quad 0 \leq r<1, \\
\int_{0}^{\infty}\left[\frac{i \nu_{2} R_{1 n}(\zeta) k_{2}^{2}}{2 \zeta^{2}-k_{2}^{2}}+Q_{2 n}(\zeta)\right] \zeta J_{n-1}(\zeta r) d \zeta=0 & r>1 \\
\int_{0}^{\infty} d \zeta \zeta\left[i G(\zeta) R_{1 n}(\zeta)-\nu_{2} Q_{2 n}(\zeta)\right] J_{n+1}(\zeta r)=-2 \alpha \beta i^{n} J_{n+1}(\alpha r), & 0 \leq r<1, \\
\int_{0}^{\infty}\left[\frac{i \nu_{2}}{2 \zeta^{2}-R_{1 n}(\zeta) k_{2}^{2}}-Q_{2 n}^{2}(\zeta)\right] \zeta J_{n+1}(\zeta r) d \zeta=0, & r>1 .
\end{array}
$$

Emboldened by our success in reducing the typographic complexity of these equations by introducing the function $G(\zeta)$, we make some further typographic simplifications by defining

$$
\begin{aligned}
& S_{n}(\zeta)=\nu_{1} P_{1 n}(\zeta) /\left(2 \zeta^{2}-k_{2}^{2}\right) \\
& L_{n}(\zeta)=i \nu_{2} R_{1 n}(\zeta) k_{2}^{2} /\left(2 \zeta^{2}-k_{2}^{2}\right)
\end{aligned}
$$


The dual integral equations may be written as

$$
\begin{array}{cc}
\int_{0}^{\infty} \zeta S_{n}(\zeta) J_{n}(\zeta r) d \zeta=A_{n} J_{n}(\alpha r)+\int_{0}^{\infty} A(\zeta) S_{n}(\zeta) J_{n}(\zeta r) d \zeta, & 0 \leq r<1, \\
\int_{0}^{\infty} S_{n}(\zeta) J_{n}(\zeta r) d \zeta=0, & r>1, \\
\int_{0}^{\infty} \zeta^{2}\left[\gamma L_{n}(\zeta)+Q_{2 n}(\zeta)\right] J_{n-1}(\zeta r) d \zeta & \\
=a_{n} J_{n-1}(\alpha r)-\int_{0}^{\infty} \zeta\left[B(\zeta) L_{n}(\zeta)+C(\zeta) Q_{2 n}(\zeta)\right] J_{n-1}(\zeta r) d \zeta, & 0 \leq r<1, \\
\int_{0}^{\infty} \zeta\left[L_{n}(\zeta)+Q_{2 n}(\zeta)\right] J_{n-1}(\zeta r) d \zeta=0, & r>1, \\
\int_{0}^{\infty}\left[\gamma L_{n}(\zeta)-Q_{2 n}(\zeta)\right] J_{n+1}(\zeta r) d \zeta & \\
=a_{n} J_{n+1}(\alpha r)-\int_{0}^{\infty} \zeta\left[B(\zeta) L_{n}(\zeta)-C(\zeta) Q_{2 n}(\zeta)\right] J_{n+1}(\zeta r) d \zeta, & 0 \leq r<1, \\
\int_{0}^{\infty} \zeta\left[L_{n}(\zeta)-Q_{2 n}(\zeta)\right] J_{n+1}(\zeta r) d \zeta=0, & r>1,
\end{array}
$$

where

$$
\begin{aligned}
A(\zeta) & =\zeta-\frac{4 \zeta^{2} \nu_{1} \nu_{2}-\left(2 \zeta^{2}-k_{2}^{2}\right)^{2}}{2\left(k_{2}^{2}-k_{1}^{2}\right) \nu_{1}}, \quad \gamma=2\left(1-\sigma^{2}\right), \quad \sigma=\frac{k_{1}}{k_{2}}<1 \\
B(\zeta) & =\frac{4 \zeta^{2} \nu_{1} \nu_{2}-\left(2 \zeta^{2}-k_{2}^{2}\right)^{2}}{\nu_{2} k_{2}^{2}}-\gamma \zeta \\
C(\zeta) & =\nu_{2}-\zeta \\
A_{n} & =2\left(\frac{\alpha^{2}}{k_{2}^{2}}-\frac{1}{2}\right) \frac{i^{n}}{\gamma} \\
a_{n} & =-2 i^{n} \alpha \beta .
\end{aligned}
$$

III. Solution to the dual integral equations. For our purposes, it is convenient to imagine the right-hand sides of (20a), (21a) and (22a) as known. Let these three expressions be $h_{n}(r), f_{n}(r), g_{n}(r)$. We must solve the dual integral equations (20) and the coupled dual integral equations (21) and (22); the solution to the first problem is independent of the solution to the second. The solutions will be obtained by introducing auxiliary functions which are defined in terms of $S_{n}(\zeta), Q_{2 n}(\zeta)$ and $L_{n}(\zeta)$. The particular form of these auxiliary functions is chosen so that (20b), (21b) and (22b) are satisfied identically. The remaining equations are then put into a suitable form for iteration in the long-wavelength approximation.

The solution proposed for (20), (21) and (22) requires $n \geq 0$ for (20) and $n>0$ for (21) and (22). This produces no difficulty since, from the integral equations, it can be seen that 


$$
\begin{aligned}
S_{-n}(\zeta) & =(-1)^{n} S_{n}(\zeta), \\
L_{-n}(\zeta) & =(-1)^{n} L_{n}(\zeta), \\
Q_{2,-n}(\zeta) & =(-1)^{n+1} Q_{2 n}(\zeta)
\end{aligned}
$$

The case $n=0$ in (21) and (22) will be treated separately.

We postulate the following solutions for (20), (21) and (22):

$$
\begin{array}{ll}
S_{n}(\zeta)=\int_{0}^{1} s_{n}(p) J_{n+1 / 2}(\zeta p)(\zeta p)^{1 / 2} d p, & n \geq 0, \\
\zeta L_{n}(\zeta)=\zeta^{1 / 2}\left[\int_{0}^{1} p_{1 n}(\eta) J_{n-1 / 2}(\zeta \eta) d \eta+\int_{0}^{1} q_{1 n}(\eta) J_{n+3 / 2}(\zeta \eta) d \eta\right], & n>0, \\
\zeta Q_{2 n}(\zeta)=\zeta^{1 / 2}\left[\int_{0}^{1} p_{2 n}(\eta) J_{n-1 / 2}(\zeta \eta) d \eta+\int_{0}^{1} q_{2 n}(\eta) J_{n+3 / 2}(\zeta \eta) d \eta\right], & n>0 .
\end{array}
$$

Only $p_{1 n}(\eta)$ will be found; the other auxiliary functions are determined similarly.

Before continuing, the following identities will be needed:

$$
\begin{array}{rlrl}
\int_{0}^{\infty} \zeta^{\mu-\nu+1} J_{\mu}(\zeta r) J_{\nu}(\zeta \eta) d \zeta & =0, & & 0<\eta<r, \nu>\mu>-1 \\
& =\frac{2^{\mu-\nu+1} r^{\mu}\left(\eta^{2}-r^{2}\right)^{\nu-\mu-1}}{\Gamma(\nu-\mu) \eta^{\nu}}, & \eta>r, \nu>\mu>-1, \\
\int_{0}^{\infty} \zeta^{\nu-\mu+1} J_{\mu}(\zeta r) J_{\nu}(\zeta \eta) d \zeta & =0, & \eta>r, \mu>\nu>-1, \\
= & \frac{2^{\nu-\mu+1} \eta^{\nu}\left(r^{2}-\eta^{2}\right)^{\mu-\nu-1}}{\Gamma(\mu-\nu) r^{\mu}}, & 0<\eta<r, \mu>\nu>-1, \\
\int_{0}^{\eta} \frac{p^{\nu+1} J_{\nu}(\zeta p)}{\left(\eta^{2}-p^{2}\right)^{1 / 2}} d p=(\pi / 2 \zeta)^{1 / 2} \eta^{\nu+1 / 2} J_{\nu+1 / 2}(\zeta \eta), & \\
& (d / d \eta)\left[\eta^{\nu} J_{\nu}(\zeta \eta)\right]=\zeta \eta^{\nu} J_{\nu-1}(\zeta \eta), \\
& (d / d \eta)\left[\eta^{-\nu} J_{\nu}(\zeta \eta)\right]=-\zeta \eta^{-\nu} J_{\nu+1}(\zeta \eta) .
\end{array}
$$

We will also want to recall that the solution to Abel's integral equation

is

$$
\int_{0}^{r} \frac{g(\eta)}{\left(r^{2}-\eta^{2}\right)^{1 / 2}} d \eta=f(r), \quad 0<r<1,
$$

$$
g(\eta)=\frac{2}{\pi} \frac{d}{d \eta} \int_{0}^{\eta} \frac{r f(r)}{\left(\eta^{2}-r^{2}\right)^{1 / 2}} d r .
$$

In Eqs. (26b, c), we have introduced four function transforms to describe the two functions $L_{n}, Q_{2 n}$. Thus we can assume two sufficient conditions among the four new functions. Eq. (22b) is satisfied if $p_{1 n}(\zeta)=p_{2 n}(\zeta)$. This can be shown if identity (27a) is applied. Eq. (21b) is also satisfied by applying (27b). This can be shown by using (27a) on the $p_{1 n}(\zeta)$ term, and $(29 \mathrm{~b})$ and $(27 \mathrm{~b})$ on the $q_{1 n}(\zeta)$ and $q_{2 n}(\zeta)$ terms. Assuming the relation

$$
q_{1 n}(\zeta)=-q_{2 n}(\zeta) / \gamma
$$


the integrand of (21a), after integration by parts, becomes

$$
\begin{aligned}
\zeta\left[\gamma L_{n}(\zeta)+Q_{2 n}(\zeta)\right]=-\zeta^{-1 / 2}(\gamma+1) & \left\{p_{1 n}(1) J_{n-3 / 2}(\zeta)\right. \\
& \left.-\int_{0}^{1} \frac{d}{d \eta}\left[\eta^{\eta-3 / 2} p_{1 n}(\eta)\right] \eta^{-n+3 / 2} J_{n-3 / 2}(\zeta \eta) d \eta\right\},
\end{aligned}
$$

where it has been assumed that $\lim _{\eta \rightarrow 0}\left[p_{1 n}(\eta) J_{n-3 / 2}(\zeta \eta)\right]=0$. Substituting (31) into (21a) and using (27a), we get

$$
\int_{0}^{r} \frac{d}{d \eta}\left\{\eta^{n-3 / 2} p_{1 n}(\eta)\right\} \frac{d \eta}{\left(r^{2}-\eta^{2}\right)^{1 / 2}}=\left(\frac{\pi}{2}\right)^{1 / 2} \frac{f_{n}(r) r^{n-1}}{\gamma+1} .
$$

The integral equation may be inverted by assuming $f_{n}(r)$ is known and using (30), (21a) and (28). The resulting equation is then integrated over $\eta$ from 0 to $\eta$ and gives

$$
p_{1 n}(\eta)=p_{1 n}^{0}(\eta)-\frac{\eta}{\gamma+1} \int_{0}^{\infty} \zeta^{1 / 2}\left[B(\zeta) L_{n}(\zeta)+C(\zeta) Q_{2 n}(\zeta)\right] J_{n-1 / 2}(\zeta \eta) d \zeta,
$$

where

$$
p_{1 n}^{0}(\eta)=\frac{a_{n}}{\gamma+1} \frac{\eta J_{n-1 / 2}(\alpha \eta)}{\sqrt{ } \alpha} .
$$

Similar calculations for (22) yield

$$
q_{1 n}(\eta)=q_{1 n}^{0}(\eta)-\frac{\eta}{\gamma(\gamma+1)} \int_{0}^{\infty} \gamma^{1 / 2}\left[B(\zeta) L_{n}(\zeta)-\gamma C(\zeta) Q_{2 n}(\zeta)\right] J_{n+3 / 2}(\zeta \eta) d \zeta,
$$

where

$$
q_{1 n}^{0}(\eta)=\frac{a_{n}}{\gamma(\gamma+1)} \frac{\eta J_{n+3 / 2}(\alpha \eta)}{\sqrt{ } \alpha} .
$$

Substituting (26b) and (26c) into (33a) and (34a) gives

$$
\begin{aligned}
& p_{1 n}(\eta)=p_{1 n}^{0}(\eta)-\frac{\eta}{\gamma+1}\left[\int_{0}^{1} p_{1 n}(t) F_{2 n}(\eta, t) d t+\int_{0}^{1} q_{1 n}(t) F_{3 n}(\eta, t) d t\right], \\
& q_{1 n}(\eta)=q_{1 n}^{0}(\eta)-\frac{\eta}{\gamma(\gamma+1)}\left[\int_{0}^{1} p_{1 n}(t) G_{1 n}(\eta, t) d t+\int_{0}^{1} q_{1 n}(t) G_{2 n}(\eta, t) d t\right],
\end{aligned}
$$

and a similar calculation for Eqs. (20) using (26a) gives

$$
s_{n}(\eta)=s_{n}^{0}(\eta)+\int_{0}^{1} F_{1 n}(\eta, t) s_{n}(t) d t
$$

where

$$
s_{n}^{0}(\eta)=A_{n}(\eta / \alpha)^{1 / 2} J_{n+1 / 2}(\alpha \eta)
$$

and

$$
F_{1 n}(\eta, t)=\left(t_{\eta}\right)^{1 / 2} \int_{0}^{\infty} A(\zeta) J_{n+1 / 2}(\zeta t) J_{n+1 / 2}(\zeta \eta) d \zeta
$$




$$
\begin{aligned}
& F_{2 n}(\eta, t)=\int_{0}^{\infty}[B(\zeta)+C(\zeta)] J_{n-1 / 2}(\zeta t) J_{n-1 / 2}(\zeta \eta) d \zeta \\
& F_{3 n}(t, \eta)= G_{1 n}(\eta, t)=\int_{0}^{\infty}[B(\zeta)-\gamma C(\zeta)] J_{n+3 / 2}(\zeta \eta) J_{n-1 / 2}(\zeta t) d \zeta \\
& G_{2 n}(\eta, t)=\int_{0}^{\infty}\left[B(\zeta)+\gamma^{2} C(\zeta)\right] J_{n+3 / 2}(\zeta \eta) J_{n+3 / 2}(\zeta t) d \zeta
\end{aligned}
$$

Due to the asymptotic behavior of $A(\zeta), B(\zeta)$ and $C(\zeta)$, Eqs. (32) may be converted to integrals over the branch cuts between $\pm k_{i}, i=1,2$. The result is

$$
\begin{gathered}
F_{1 n}(\eta, t)=i(t \eta)^{1 / 2} \frac{k_{2}^{2}}{2 \gamma}\left\{\int_{0}^{1} d y 4 y^{2}\left(1-y^{2}\right)^{1 / 2} H_{n+1 / 2}^{(1)}\left(t_{>} k_{2} y\right) J_{n+1 / 2}\left(t_{<} k_{2} y\right)\right. \\
\left.\quad+\int_{0}^{1} d y \frac{\left(2 \sigma^{2} y^{2}-1\right)^{2}}{\left(1-y^{2}\right)^{1 / 2}} H_{n+1 / 2}^{(1)}\left(t_{>} \sigma k_{2} y\right) J_{n+1 / 2}\left(t_{<} \sigma k_{2} y\right)\right\} \\
F_{2 n}(\eta, t)=-4 i \sigma^{4} k_{2}^{2} \int_{0}^{1} d y y^{2}\left(1-y^{2}\right)^{1 / 2} H_{n-1 / 2}^{(1)}\left(\sigma k_{2} t_{>} y\right) J_{n-1 / 2}\left(\sigma k_{2} t_{<} y\right) \\
-i k_{2}^{2} \int_{0}^{1} d y\left[\frac{\left(2 y^{2}-1\right)^{2}}{\left(1-y^{2}\right)^{1 / 2}}+\left(1-y^{2}\right)^{1 / 2}\right] H_{n-1 / 2}^{(1)}\left(k_{2} t_{>} y\right) J_{n-1 / 2}\left(k_{2} t_{<} y\right)
\end{gathered}
$$

$$
G_{2 n}(\eta, t)=-4 i \sigma^{4} k_{2}^{2} \int_{0}^{1} d y y^{2}\left(1-y^{2}\right)^{1 / 2} H_{n+3 / 2}^{(1)}\left(\sigma k_{2} t_{>} y\right) J_{n+3 / 2}\left(\sigma k_{2} t_{<} y\right)
$$$$
-i k_{2}^{2} \int_{0}^{1} \frac{d y}{\left(1-y^{2}\right)^{1 / 2}}\left[\left(2 y^{2}-1\right)^{2}+\gamma^{2}\left(1-y^{2}\right)\right] H_{n+3 / 2}^{(1)}\left(k_{2} t_{>} y\right) J_{n+3 / 2}\left(k_{2} t_{<} y\right),
$$

$$
\begin{gathered}
F_{3 n}(\eta, t)=G_{1 n}(t, \eta)=-4 i \sigma^{4} k_{2}^{2} \int_{0}^{1} d y y^{2}\left(1-y^{2}\right)^{1 / 2} H_{n-1 / 2}^{(1)}\left(\sigma k_{2} \eta y\right) J_{n+3 / 2}\left(\sigma k_{2} t y\right) \\
-i k_{2}^{2} \int_{0}^{1} \frac{d y}{\left(1-y^{2}\right)^{1 / 2}}\left[\left(2 y^{2}-1\right)^{2}\right. \\
\left.-\gamma\left(1-y^{2}\right)\right] H_{n-1 / 2}^{(1)}\left(k_{2} \eta y\right) J_{n+3 / 2}\left(k_{2} t y\right), \quad t<\eta, \\
F_{3 n}(\eta, t)=G_{1 n}(t, \eta)=-4 i \sigma^{4} k_{2}^{2} \int_{0}^{1} d y y^{2}\left(1-y^{2}\right)^{1 / 2} H_{n+3 / 2}^{(1)}\left(\sigma k_{2}^{2} t y\right) J_{n-1 / 2}\left(\sigma k_{2} \eta y\right)
\end{gathered}
$$

$$
\begin{aligned}
& -4 i k_{2}^{2} \int_{0}^{1} d y y^{2} \frac{\left(y^{2}-1\right)}{\left(1-y^{2}\right)^{1 / 2}} H_{n+3 / 2}^{(1)}\left(k_{2} t y\right) J_{n-1 / 2}\left(k_{2} \eta y\right) \\
& -\frac{i \eta^{n-1 / 2}}{t^{n+1 / 2}}(2 n+1), \quad t>\eta .
\end{aligned}
$$

We have used the notation

$$
\underset{<}{t>}=\begin{aligned}
& t \\
& \eta
\end{aligned} \text { if } t>\eta \quad \text { and } \quad \underset{<}{t_{>}}=\begin{aligned}
& \eta \\
& t
\end{aligned} \text { if } t<\eta .
$$

If these kernels are expanded in powers of $k_{1}, k_{2}$, it can be shown that $F_{1 n}(\eta, t)$, $F_{2 n}(\eta, t)$ and $G_{2 n}(\eta, t)$ are of the order $k^{2}$ and $F_{3 n}(\eta, t)$ and $G_{1 n}(\eta, t)$ are of the order of $k^{4}$. The solutions to (35) and (36) are obtained by iteration in which the first-order solution is $p_{1 n}(\eta)=p_{1 n}^{0}(\eta), q_{1 n}(\eta)=q_{1 n}^{0}(\eta)$ and $s_{n}(\eta)=s_{n}^{0}(\eta)$. 
To obtain the complete solution, we must now solve (17) and (18) for the case $n=0$. The equations under these circumstances decouple into

$$
\begin{gathered}
\int_{0}^{\infty} \zeta L_{0}(\zeta) J_{1}(\zeta r) d \zeta=0, \quad r>1, \\
\int_{0}^{\infty} \zeta A^{\prime}(\zeta) L_{0}(\zeta) J_{1}(\zeta r) d \zeta=-2 \alpha \beta J_{1}(\alpha r), \quad 0 \leq r<1, \\
\int_{0}^{\infty} Q_{10}(\zeta) J_{1}(\zeta r) d \zeta=0, \quad r>1, \\
\int_{0}^{\infty} \nu_{2} Q_{10}(\zeta) J_{1}(\zeta r) d \zeta=0, \quad 0 \leq r<1,
\end{gathered}
$$

where

$$
A^{\prime}(\zeta)=\frac{4 \zeta^{2} \nu_{1} \nu_{2}-\left(2 \zeta^{2}-k_{2}^{2}\right)^{2}}{\nu_{2} k_{2}^{2}}
$$

(40) implies $Q_{10}(\zeta)=0$. To solve (39), let

$$
L_{0}(\zeta)=\zeta^{-1 / 2} \int_{0}^{1} p_{0}(\eta) J_{3 / 2}(\zeta \eta) d \eta
$$

Proceeding as before, (41) identically satisfies (39b). (39a) may be put in the form

$$
\gamma \int_{0}^{\infty} \zeta^{2} L_{0}(\zeta) J_{1}(\zeta r) d \zeta=-2 \alpha \beta J_{1}(\alpha r)-\int_{0}^{\infty} B(\zeta) \zeta L_{0}(\zeta) J_{1}(\zeta r) d \zeta .
$$

Using (41) in (42), and inverting by using Abel's solution, we get

$$
p_{0}(\eta)=-\frac{1}{\gamma} 2 \alpha \beta \eta \frac{J_{3 / 2}(\alpha \eta)}{\sqrt{ } \alpha}-\frac{\eta}{\gamma} \int_{0}^{1} d t p_{0}(t) F_{0}(\eta, t),
$$

where

$$
\begin{aligned}
F_{0}(\eta, t)= & \int_{0}^{\infty} d \zeta B(\zeta) J_{3 / 2}(\zeta t) J_{3 / 2}(\zeta \eta) \\
= & -4 i \sigma^{4} k_{2}^{2} \int_{0}^{1} d y y^{2}\left(1-y^{2}\right)^{1 / 2} H_{3 / 2}^{(1)}\left(\sigma k_{2} t_{>} y\right) J_{3 / 2}\left(\sigma k_{2} t_{<} y\right) \\
& -i k_{2}^{2} \int_{0}^{1} d y \frac{\left(2 y^{2}-1\right)^{2}}{\left(1-y^{2}\right)^{1 / 2}} H_{3 / 2}^{(1)}\left(k_{2} t_{>} y\right) J_{3 / 2}\left(k_{2} t_{<} y\right), \\
F_{0}(\eta, t)= & 0\left(k^{2}\right) .
\end{aligned}
$$

IV. Effective elastic modulus. Having solved the problem of the scattering of incident plane $P$-waves on a single crack, we procecd to find the effective elastic modulus associated with the scattering of a $P$-wave by a random distribution of such cracks, randomly oriented as well, and imbedded in an otherwise elastic material. The effective modulus will be calculated by considering the flawed material as an equivalent homogeneous isotropic elastic material subjected to a constant traction on the surface. The deformational energy of the equivalent homogeneous material will be calculated and set equal to the deformational energy of the flawed material. It will be assumed that 
the density of inhomogeneities is small, so that in the lowest approximation the deformational energy of the flawed system is equal to the energy of the unflawed homogeneous host matrix plus a correction due to the flaws.

We write the total energy in the flawed case as

$$
E=E^{0}+\Delta E=\frac{1}{2} \int_{0} \tau_{i j}^{0} \epsilon_{i j}^{0} d v+\frac{1}{2} \int_{.}\left(\tau_{i j} \epsilon_{i j}-\tau_{i j}^{0} \epsilon_{i j}^{0}\right) d v,
$$

where the first integral is evidently the result in the unflawed case. In these expressions, $v$ is the total volume. The volume $v$ in the flawed case is bounded by an exterior surface $S$ and the surfaces of the cracks $S_{0}$. By an energetic application of the divergence theorem, the boundary conditions $\tau_{n i}=0$ on $S_{0}$ where $n$ is the normal to $S_{0}$, and the reciprocity theorem, we can write (45) as

$$
E=\frac{1}{2} \int_{0} \tau_{i j}^{0} \epsilon_{i j} d v=\frac{1}{2} \int_{0} \tau_{i j}^{0} \epsilon_{i j}^{0} d v+\frac{1}{2} \int_{S_{0}} \tau_{i j}^{0} n_{j}\left[u_{i}\right] d S
$$

where $\left[u_{i}\right]$ is the jump in $u_{i}$ across $S_{0}$.

Let the effective moduli be $\lambda_{1}, \mu_{1}$ and the elastic moduli for the matrix material be $\lambda, \mu$. There exist two Hookean operators $L_{1}$ and $L_{2}$ connecting the stress and the strain:

$$
\epsilon_{i j}=L_{1}\left(\tau_{i j}^{0}\right), \quad \epsilon_{i j}^{0}=L_{0}\left(\tau_{i j}^{0}\right) .
$$

Thus (46) becomes

$$
\tau_{i j}^{0} L_{1}\left(\tau_{i j}^{0}\right) v=\tau_{i j}^{0} L_{0}\left(\tau_{i j}^{0}\right) v+\sum_{p=1}^{N} \tau_{i j}^{0} \int_{S_{p}} n_{i}\left[u_{i}\right] d S,
$$

where the two volume integrals have been evaluated by virtue of the uniformity of the fields in both cases. The surface $S_{0}$ has been broken into $N$ fragments corresponding to each of the $N$ disks in $v$; each separate disk is identified by a surface $S_{\mathfrak{p}}$. Each of the $S_{p}$ is randomly oriented and uniformly distributed throughout the material.

The jump in displacement $\left[u_{i}\right]$ must be determined and the static limit taken. The latter step requires the calculation of the lowest-order terms of $\left[u_{\imath}\right]$. The discontinuity in the $z$-component of the displacement across a circular crack is

$$
\left[u_{z}\right]=2 k_{2}^{2} \sum_{n=-\infty}^{\infty} \exp (i n \phi) \int_{0}^{\infty} S_{n}(\zeta) J_{n}(\zeta r) d \zeta
$$

from Eqs. (4c), (7a, b), (12b, c) and (19a). We substitute from Eq. (26a) for $S_{n}(\zeta)$ and interchange the order of integration. We get

$$
\left[u_{z}\right]=4\left(\frac{2}{\pi}\right)^{1 / 2} k_{2}^{2} \sum_{n=0}^{\infty} r^{n} \epsilon_{n} \cos n \phi \int_{r}^{1} d p \frac{s_{n}(p)}{\left(p^{2}-r^{2}\right)^{1 / 2} p^{n}}
$$

where

$$
\begin{array}{rlrl}
\epsilon_{n} & =\frac{1}{2}, & & n=0, \\
& =1, & n \neq 0 .
\end{array}
$$

The dominant term in the sum is that for $n=0$. Thus the jump in $u_{\mathbf{z}}$ in the static limit is

$$
\left[u_{z}\right]_{S L} \cong \frac{4}{\pi} A_{0} k_{2}^{2}\left\{\left(1-r^{2}\right)^{1 / 2}\right\}
$$


from (36b). Using a similar procedure for the jump in $u_{r}$ and $u_{\phi}$, we have

$$
\begin{aligned}
& {\left[u_{r}\right]=-4 i\left(\frac{2}{\pi}\right)^{1 / 2} \sum_{n=1}^{\infty} \cos n \phi\left[\int_{r}^{1} \frac{d \eta p_{n}(\eta) r^{n-1}}{\eta^{n-1 / 2}\left(\eta^{2}-r^{2}\right)^{1 / 2}}\right.}
\end{aligned}
$$

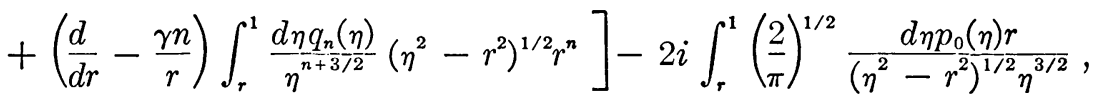

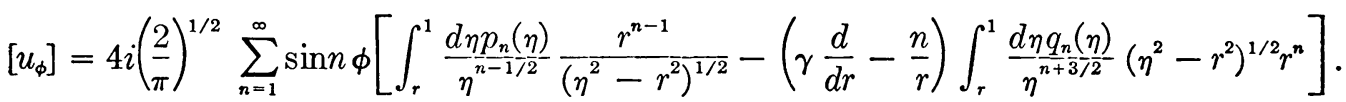

The dominant terms are for the case $n=1$. The results are

$$
\begin{aligned}
& {\left[u_{\phi}\right]_{1}=+(16 \alpha \beta /(1+\gamma) \pi)\left\{\left(1-r^{2}\right)^{1 / 2}\right\},} \\
& {\left[u_{r}\right]_{1}=-(16 \alpha \beta /(\gamma+1) \pi)\left\{\left(1-r^{2}\right)^{1 / 2}\right\},}
\end{aligned}
$$

from (35a) and (35b).

The stress $\tau_{i i}^{0}$ is obtained from the incident field

$$
\Phi^{0}=\exp \left(i\left[\alpha r \cos \left(\phi-\phi_{0}\right)+\beta^{2}\right]\right) .
$$

The Hookean operator acting on the stress $\tau_{i j}^{0}$ is

$$
\epsilon_{i j}=L_{1}\left(\tau_{i j}\right)=\frac{\tau_{i j}}{2 \mu_{1}}-\frac{\lambda_{1} \delta_{i j} \tau_{k k}}{2 \mu_{1}\left(2 \mu_{1}+3 \lambda_{1}\right)} .
$$

The energy density in the equivalent unflawed medium is

$$
\tau_{i j}^{0} L_{1}\left(\tau_{i j}^{0}\right)=\left(\lambda_{1}+2 \mu_{1}\right) k_{1}^{4}\left(\Phi^{0}\right)^{2} .
$$

The scalar quantity $\int_{S_{0}} \tau_{i j}^{0} n_{j}\left[u_{i}\right] d S$ is independent of choice of coordinates. Hence, we take a coordinate system such that the normal of $S_{p}$ is in the $z$-direction for any disk. The stresses in the incident field are

$$
\begin{aligned}
\tau_{r z}^{0} & =-2 \mu \alpha \beta \cos \left(\phi-\phi_{0}\right) \Phi^{0}, \\
\tau_{\phi z}^{0} & =2 \mu \alpha \beta \sin \left(\phi-\phi_{0}\right) \Phi^{0}, \\
\tau_{z z}^{0} & =-\left(\lambda k_{1}^{2}+2 \mu \beta^{2}\right) \Phi^{0} .
\end{aligned}
$$

To this point, we have considered the scattering by a crack of unit radius. We now dimensionalize the result for a crack of radius $a$, and we have

$$
\begin{aligned}
& {\left[u_{z}\right]=\frac{4}{\pi} A_{0} k_{2}^{2} a^{2}\left(a^{2}-r^{2}\right)^{1 / 2},} \\
& {\left[u_{r}\right]=-\frac{16 \alpha \beta a^{2}\left(a^{2}-r^{2}\right)^{1 / 2}}{(1+\gamma) \pi} \cos \left(\phi-\phi_{0}\right),} \\
& {\left[u_{\phi}\right]=\frac{16 \alpha \beta a^{2}}{(1+\gamma) \pi}\left(a^{2}-r^{2}\right)^{1 / 2} \sin \left(\phi-\phi_{0}\right) .}
\end{aligned}
$$

Substituting (56), (57) and (58) into (48) gives

$$
\frac{1}{\lambda_{1}+2 \mu_{1}}=\frac{1}{\lambda+2 \mu}\left\{1+\frac{8}{3} \frac{a^{3}}{v} \sum_{i}\left[\frac{8 \mu \sin ^{2} \theta_{0}^{i} \cos ^{2} \theta_{0}^{i}}{3 \lambda+4 \mu}+\frac{\left(\lambda+2 \mu \cos ^{2} \theta_{0}^{i}\right)^{2}}{2 \mu(\lambda+\mu)}\right]\right\} \text {. }
$$


It is now elementary to perform an average of this expression over all possible angles on the unit sphere. We get

$$
\left\langle\frac{1}{\lambda_{1}+2 \mu_{1}}\right\rangle=\frac{1}{\lambda+2 \mu}\left\{1+\frac{8}{3} \frac{N a^{3}}{v}\left[\frac{16}{15} \frac{\mu}{3 \lambda+4 \mu}+2 \frac{\frac{1}{3} \lambda+\frac{1}{5} \mu}{\lambda+\mu}+\frac{1}{2} \frac{\lambda^{2}}{\mu(\lambda+\mu)}\right]\right\}
$$

as the expectation value; i.e., the average compressional modulus of the flawed material in terms of the properties of the matrix, i.e. the unflawed material, and $\mathrm{Na}^{3} / v$, the number of cracks per unit volume scaled by $a^{3}$, a quantity having the dimensions of volume derived from the radius of the crack.

\section{References}

[1] T. T. Wu, The effect of inclusion shape on the elastic moduli of a two-phase material, Int. J. Solids Structures 2, 1-8 (1966)

[2] J. B. Walsh, Attenuation in partially melted material, J. Geophys. Res. 73, 2209-2216 (1968)

[3] J. B. Walsh, New analysis of attenuation in partially melted rock, J. Geophys. Res. 74, 4333-4337 (1969)

[4] A. K. Mal and L. Knopoff, Elastic wave velocities in two-component systems, J. Inst. Maths. Applics. 3, 376-387 (1967)

[5] D. D. Ang and L. Knopoff, Diffraction of vector elastic waves by a clamped finite strip, Proc. Nat. Acad. Sciences 52, 201-207 (1964)

[6] D. D. Ang and L. Knopoff, Diffraction of vector elastic waves by a finite crack, Proc. Nat. Acad. Sciences 52, 1075-1081 (1964)

[7] A. K. Mal, D. D. Ang and L. Knopoff, Diffraction of elastic waves by a rigid circular disc, Proc. Camb. Phil. Soc. 64, 237-247 (1968) 